\title{
Reconfigurable Magneto-Electric Dipole Antennas for Base Stations in Modern Wireless Communication Systems
}

\author{
Lei Ge $\mathbb{D}^{1},{ }^{1}$ Xujun Yang, ${ }^{1}$ Zheng Dong, ${ }^{1}$ Dengguo Zhang $\mathbb{D},{ }^{1}$ and Xierong Zeng ${ }^{2}$ \\ ${ }^{1}$ College of Electronic Science and Technology, Shenzhen University, Shenzhen, China \\ ${ }^{2}$ College of Material Science and Engineering, Shenzhen University, Shenzhen, China \\ Correspondence should be addressed to Lei Ge; leige@szu.edu.cn
}

Received 19 January 2018; Accepted 25 March 2018; Published 15 May 2018

Academic Editor: Luca De Nardis

Copyright (c) 2018 Lei Ge et al. This is an open access article distributed under the Creative Commons Attribution License, which permits unrestricted use, distribution, and reproduction in any medium, provided the original work is properly cited.

\begin{abstract}
Magneto-electric (ME) dipole antennas, with the function of changing the antenna characteristics, such as frequency, polarization, or radiation patterns, are reviewed in this paper. The reconfigurability is achieved by electrically altering the states of diodes or varactors to change the surface currents distributions or reflector size of the antenna. The purpose of the designs is to obtain agile antenna characteristics together with good directive radiation performances, such as low cross-polarization level, high front-to-back ratio, and stable gain. By reconfiguring the antenna capability to support more than one wireless frequency standard, switchable polarizations, or cover tunable areas, the reconfigurable ME dipole antennas are able to switch functionality as the mission changes. Therefore, it can help increase the communication efficiency and reduce the construction cost. This shows very attractive features in base station antennas of modern wireless communication applications.
\end{abstract}

\section{Introduction}

Owing to the tremendous evolution of wireless communications, the electromagnetic frequency spectrum is more and more crowded and the communication environment is more and more complex. To improve the communication quality, the fifth-generation $(5 \mathrm{G})$ mobile communications were proposed several years ago and will be commercially available in early 2020. In 5G mobile communications, higher transmission speed, higher reliability, and lower delay are required.

There are three ways to increase the communication capacity as shown in Figure 1: (1) using new frequency bands with wider bandwidth; (2) improving the spectrum efficiency; (3) using more radio frequency (RF) cells with smaller size. Because of this, in $5 \mathrm{G}$ mobile communications, millimeter-wave bands will be used to increase the frequency bandwidth; multi-input multi-output (MIMO) antennas will be applied to increase the network density. In the past several years, reconfiguration technique applied in RF systems to effectively increase the spectrum efficiency has been demonstrated. Accordingly, reconfiguration technique with the ability to improve the frequency spectrum utilization has aroused great research interest in both industrial and academic areas. As the front end, antennas play a very important role in any wireless communication system. Antennas with reconfigurable characteristics can effectively enhance the system performance because they are able to adjust the antenna characteristics automatically and make them suitable to complex scenarios. Antennas with the function to dynamically change their operating frequency, polarization, or radiation patterns are needed to improve the channel capacity in communication systems. According to Friis transmission equation,

$$
\frac{P_{R}}{P_{T}}=\left(1-\left|\Gamma_{T}\right|^{2}\right)\left(1-\left|\Gamma_{R}\right|^{2}\right) G_{T} G_{R}\left|\widehat{\rho}_{T} \cdot \widehat{\rho}_{R}\right|^{2}\left(\frac{\lambda}{4 \pi r}\right)^{2} .
$$

The transmission efficiency between the transmitter and receiver mainly depends on three factors: impedance matching $\left(\Gamma_{T}, \Gamma_{R}\right)$, antenna gain $\left(G_{T}, G_{R}\right)$, and polarization matching $\left(\widehat{\rho}_{T}, \hat{\rho}_{R}\right)$. Therefore, reconfigurable antennas can be basically categorized into three types: (1) frequency reconfigurable antennas [1-5]; (2) polarization reconfigurable antennas [6-11]; (3) pattern reconfigurable antennas [5, 12-18]. 


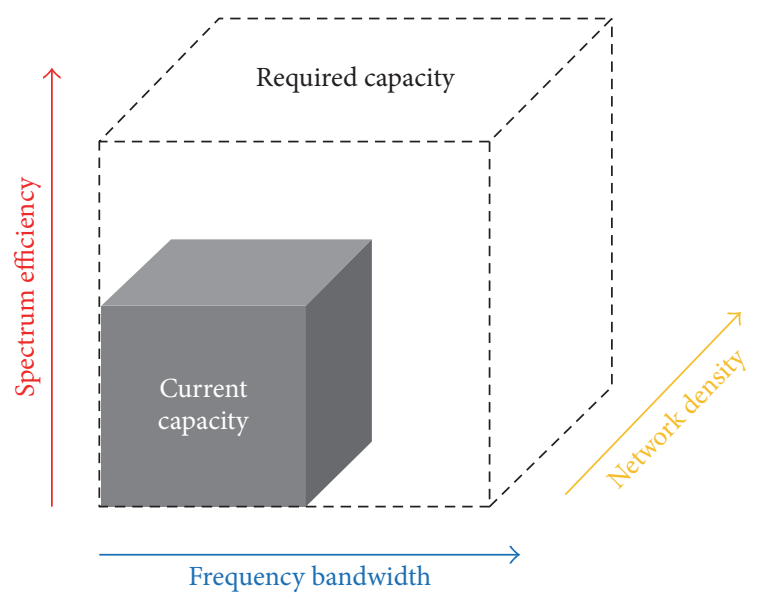

FIGURE 1: Methods for increasing the capacity.

Although many reconfigurable antennas have been proposed, they are usually designed based on microstrip structures $[1,3-5,9-13,15,16]$. Due to the narrow bandwidth, they are not appropriate to be applied in base stations. On the other hand, along with the development of wireless communications, antennas with wider bandwidth and good direction radiation patterns are required. Therefore, the conventional directional antenna candidates cannot satisfy the need of reconfigurable base station antennas.

In 2006, Prof. Luk invented a new antenna type specified as the magneto-electric (ME) dipole antenna [19], which consists of a magnetic dipole and an electric dipole. By exciting the complementary dipoles with suitable amplitudes and phases simultaneously, the antenna is able to produce good radiation characteristics over a wide frequency band. Taking advantages of the ME dipole antennas, they are very appreciated for the extensive applications of mobile cellular networks. When combining the ME dipole with reconfiguration technique, consequent reconfigurable ME dipole antennas can be developed for base stations in modern wireless systems. In this paper, three different kinds of reconfigurable ME dipole antennas are reviewed, namely, frequency reconfigurable ME dipole, polarization reconfigurable ME dipole, and beamwidth reconfigurable ME dipoles. In Section 2, a frequency reconfigurable ME dipole antenna is reviewed. In Section 3, several polarization switchable ME dipole antennas are reviewed. In Section 4, three beamwidth reconfigurable ME dipole antennas are reviewed. These designs show attractive features for modern wireless communication systems.

\section{Frequency Reconfigurable ME Dipole Antenna}

In this section, we depict a frequency reconfigurable $\mathrm{ME}$ dipole antenna [2]. Figure 2 gives the configuration of the antenna. The wide-narrowband antenna reconfiguration is demonstrated by systematically incorporating a broadband ME dipole antenna and a frequency reconfigurable narrowband dipole antenna. The ME dipole, as a part of the overall antenna structure, plays a crucial role in providing wideband operation. On the other hand, a length-switchable directed dipole antenna is designed to feature reconfigurable narrowband operation. A vertically oriented balun is used to feed the dipole. By using a box-shaped cavity, the dipole can generate good unidirectional radiation performance. By dynamically switching the states of PIN diodes embedded in the thin dipole, a changeable effective length is achieved. Besides, the surface current flowed on the thin dipole and the ME dipole can be changed by five groups of switches. Therefore, the antenna can be switched between four narrowband modes and a wideband mode. Notably, when the thin dipole is switched OFF, the ME dipole radiates with a wide band that can cover the operating frequency of the four narrow bands.

The measured reflection coefficients are presented in Figure 3. As observed from the figure, the fractional impedance bandwidth of the wideband mode is $89 \%$ and four different narrow bands appear at $0.95,1.35,1.7$, and $2 \mathrm{GHz}$, respectively. In addition, the main beam of the radiation patterns is always fixed in the broadside direction with front-to-back ratios of above $20 \mathrm{~dB}$ and cross-polarization levels of below $-22 \mathrm{~dB}$. Therefore, the design can work in one wideband mode for sensing and four reconfigurable narrowband modes for communications, which is attractive for base stations in cognitive radio.

\section{Polarization Reconfigurable ME Dipole Antenna}

In this section, we present three polarization reconfigurable ME dipole antennas. All the three designs [6-8] are based on similar four-sectional ME dipole geometry, while the polarization reconfiguration is realized by different feed structures and switching methods. The based ME dipole structure is based on the designs in $[20,21]$.

In [6], a feeding structure of an end-curving cross dipole for excitation of the polarization diversity antenna is proposed, as shown in Figure 4(a). PIN diodes are embedded into the arms of the cross dipole. Diodes can be switched $\mathrm{ON}$ or $\mathrm{OFF}$ in real time; therefore the RF signals can be coupled from the coaxial cable to the ME dipole. Accordingly, a polarization diversity between horizontal and vertical polarization modes can be produced. An overlapped impedance bandwidth ranging from 1.86 to $2.35 \mathrm{GHz}$ is achieved at both orthogonally polarized states. Furthermore, the antenna presents a good radiation performance with an $8 \mathrm{dBi}$ gain over the whole band.

In [7], another ME dipole antenna with the diversity of polarization is presented, which can operate between one linear polarization (LP) and two orthogonal circular polarizations (CP). In this design, four PIN diodes are embedded into the thin line printed on the diagonal position of four horizontal metal plates for controlling connection or disconnection. When all diodes are in OFF, LP operation is achieved, which means no thin lines are connected. To realize CP radiation, one single thin line is connected at the diagonal parts, perturbation is therefore introduced, and then corresponding left-handed circularly polarization (LHCP) or right-handed circularly polarization (RHCP) can emerge. The proposed design possesses admirable features such as gain stability and good directional radiation patterns. 


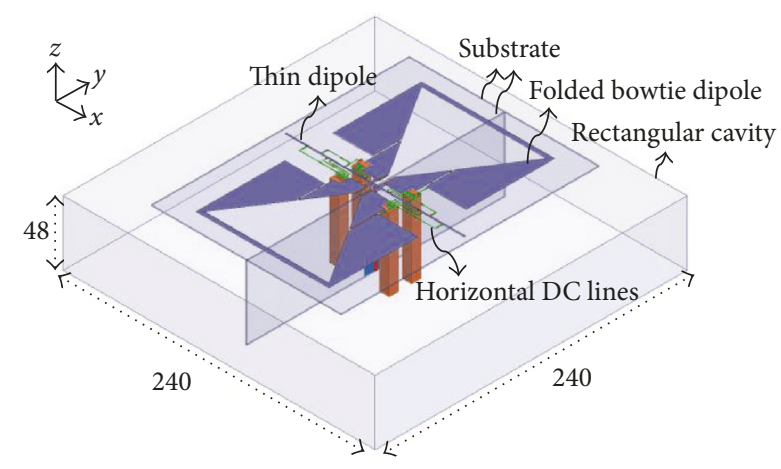

(a)

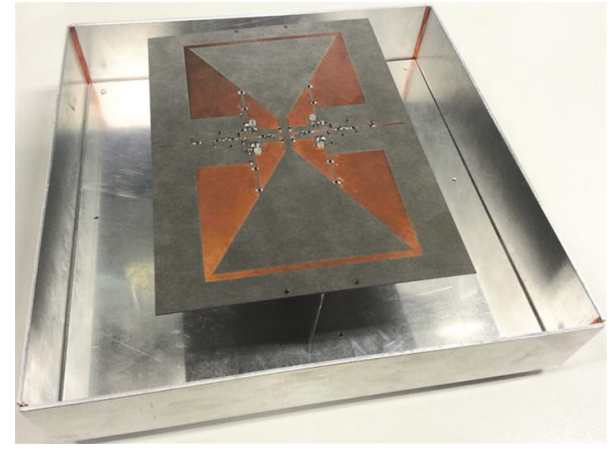

(b)

Figure 2: Geometry of the frequency reconfigurable ME dipole antenna [2]: (a) 3D view of the design; (b) fabricated prototype.

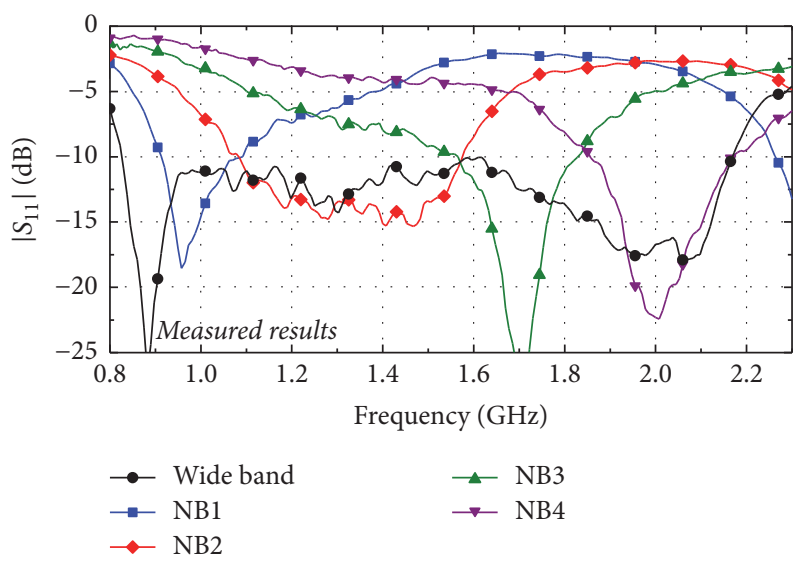

FIGURE 3: Measured $\left|S_{11}\right|$ of the frequency reconfigurable ME dipole antenna.

In [8], the antenna is composed of an ME dipole, a substrate-integrate waveguide (SIW) cavity, and some DC lines as given in Figure 5. The design is comprised of four square metallic patches, four vertically oriented metal posts, and a strip. In this design, two sets of PIN diodes (SW1 and SW2) are used to help connecting or disconnecting the diagonal metal patches with the strip. By controlling the states of the two pairs of switches, one LP and two CP modes can be switched as depicted in Figure 6. When all diodes are $\mathrm{ON}$, LP radiation can emerge. To excite $\mathrm{CP}$ modes, one of the two diode groups is ON while another is OFF. Hence, three polarization states can be realized by controlling the states of the switches. The effective overlapped bandwidth is $16 \%$ covering $5.07-5.95 \mathrm{GHz}$ for applications of $5 \mathrm{G} \mathrm{WiFi}$. The measured antenna gain maintains stability at approximately $8.2 \mathrm{dBi}$ across the band of interest for all operation states.

\section{Beamwidth Reconfigurable ME Dipole Antenna}

As discussed, frequency reconfigurable and polarization reconfigurable antennas can be designed on the basis of the ME dipole. In this section, we will discuss radiation pattern reconfiguration based on the ME dipole structure. Conventionally, pattern reconfigurable antennas are mainly designed to switch the radiating direction of the antenna. Furthermore, an antenna, with the ability of electronically controlling its radiation beamwidth, can enhance the communication quality of wireless systems. Thus, beamwidth reconfigurable antennas are demanded for modern base stations. In this section, three different methods are introduced to implement beamwidth reconfiguration of $\mathrm{ME}$ dipole antennas.

First, a three-element linear ME dipole array is used to achieve tunable beamwidth [17]. As shown in Figure 7, the linear array is composed of three ME dipoles and a feeding network. The feeding network is able to reset the phase distribution with a power distribution ratio of $1: 2: 1$ for three antenna elements. The phase difference between Antennas 1 and 3 and Antenna 2 is $\beta=0^{\circ} / 50^{\circ} / 108^{\circ}$. The operation principle of the antenna can be explained by a simplified three-element linear array. As illustrated in Figure 8, the three elements are located along the $y$-axis and the space between them is $d$. In this condition, it is assumed that the three antenna elements are all the same and well-isolated. The radiation field in the yoz-plane can be expressed as

$$
\begin{aligned}
F & (\theta)_{T} \\
& =\left[a_{1} e^{-j\left(k r_{1}+\psi_{1}\right)}+a_{2} e^{-j\left(k r_{2}+\psi_{2}\right)}+a_{3} e^{-j\left(k r_{3}+\psi_{3}\right)}\right] f(\theta),
\end{aligned}
$$

where the amplitudes and phases of excitations are $a_{n}$ and $\psi_{n}(n=1,2,3)$ and $f(\theta)$ is the radiation pattern of a single antenna element. In order to achieve symmetrical radiation pattern in the $H$-plane, the amplitudes and phases of Ant 1 and 3 are set to be the same. Assuming $a_{2}=M a_{1}=M a_{3}$, $\psi_{2}=\psi_{1}+\beta=\psi_{3}+\beta$, and the factor of the antenna array is decreased to

$$
A F=a_{1}[2 \cos (k d \sin \theta)+M \cos \beta-j M \sin \beta] .
$$

For different $\beta$, the array factor in the $y o z$-plane is given in Figure 9 with $d=0.5 \lambda_{0}$ and $M=\sqrt{2}$. Especially, by utilizing the variation of the beam of the array factor and then multiplying the array factor with the pattern of a single element, the reconfiguration of the beamwidth is able to be realized. 


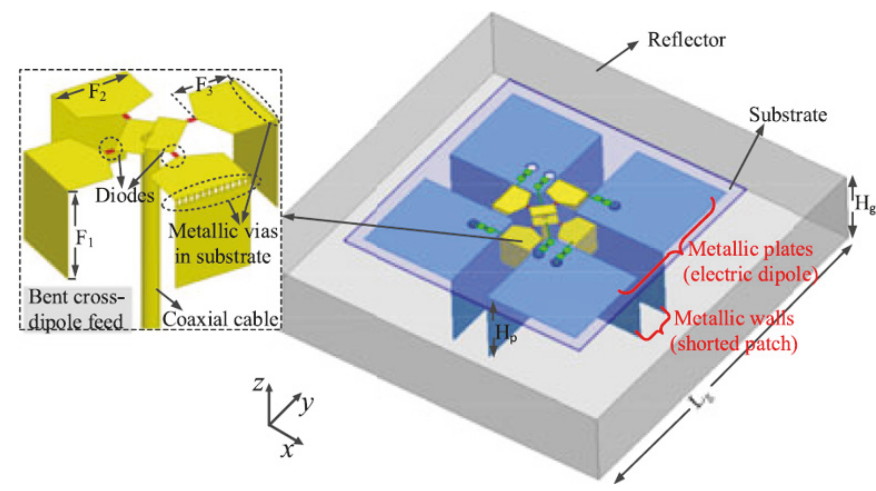

(a)

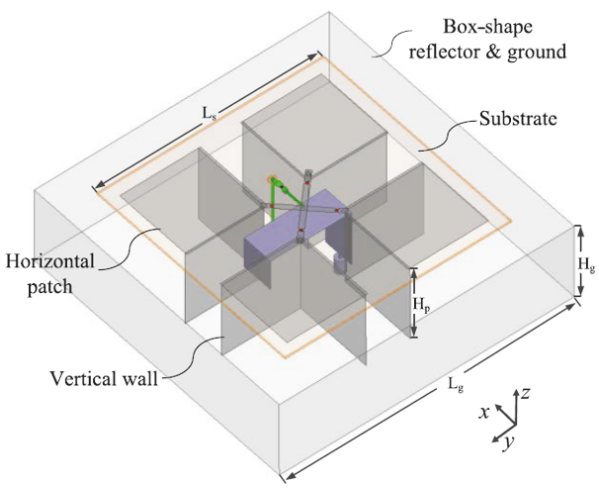

(b)

FiguRE 4: Geometry of the polarization reconfigurable ME dipole antennas: (a) the antenna in [6]; (b) the antenna in [7].

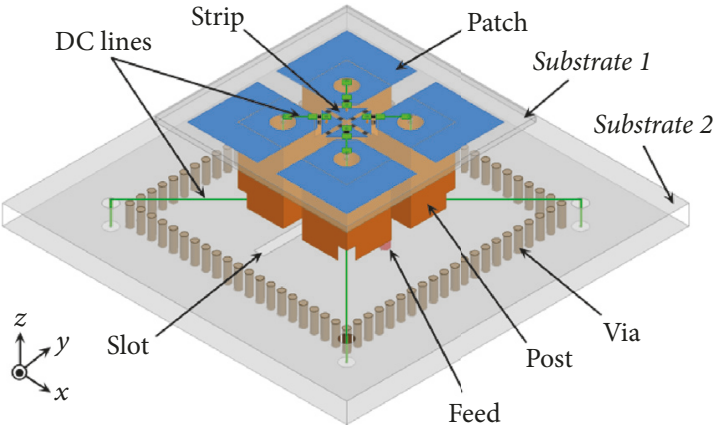

(a)

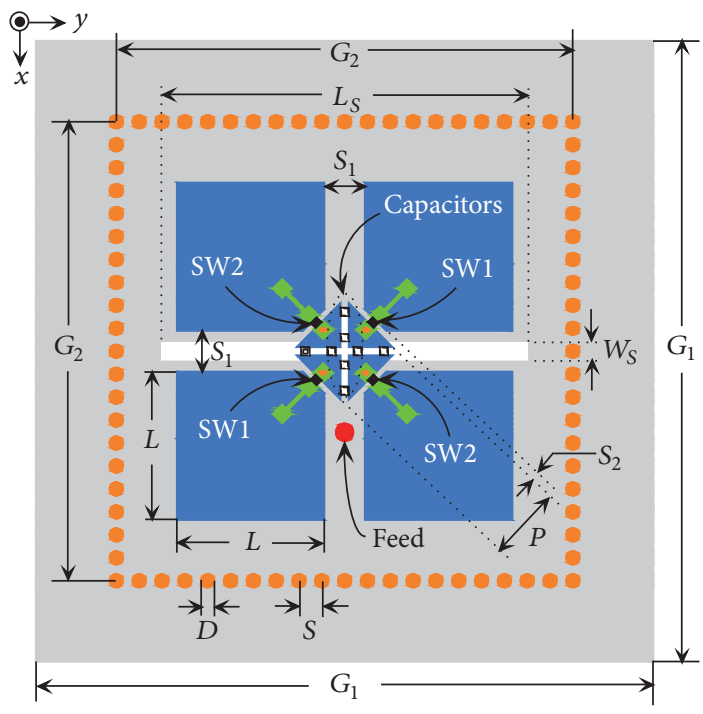

(b)

FIGURE 5: Geometry of the polarization reconfigurable ME dipole antenna [8]: (a) 3D view; (b) top view.

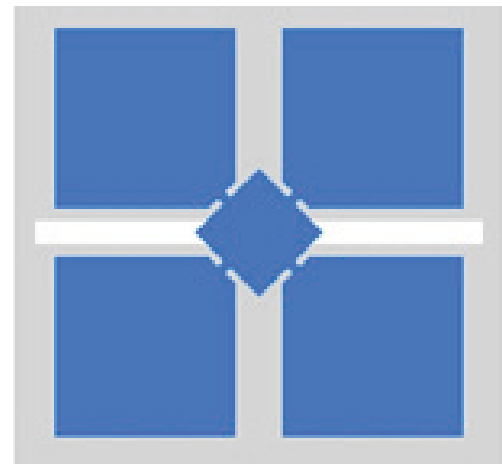

(a)

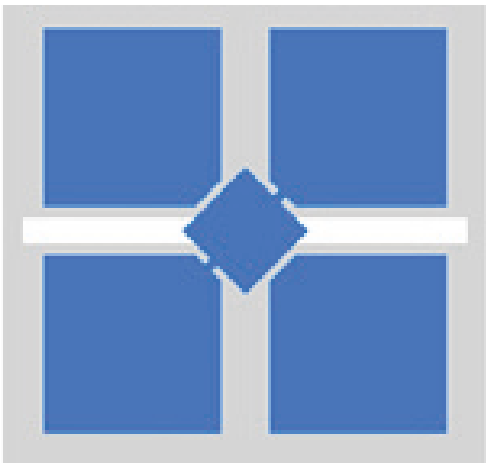

(b)

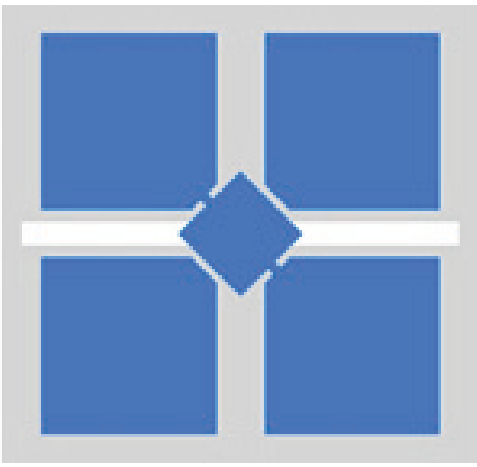

(c)

FIGURE 6: Switching states of the polarization reconfigurable ME dipole antenna [8]: (a) LP state; (b) RHCP state; (c) LHCP state. 


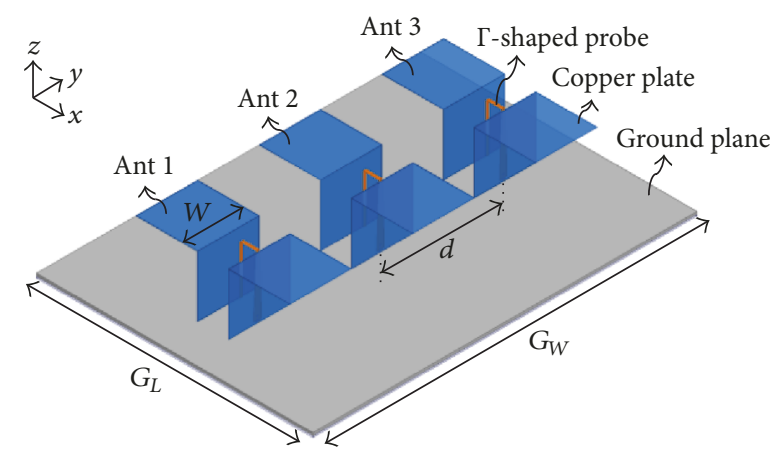

FIGURE 7: Geometry of the three-element linear ME dipole array with beamwidth reconfiguration.

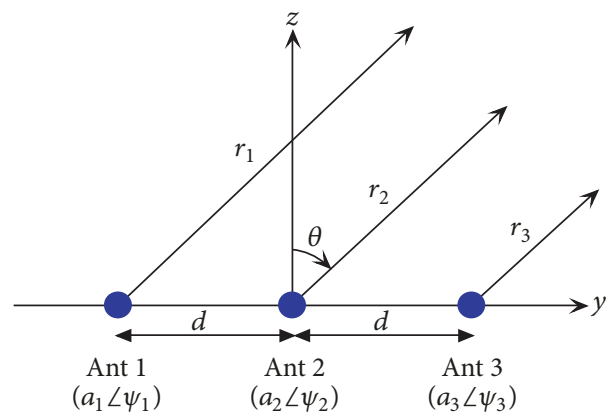

FIGURE 8: Three-element array model.

Secondly, LP and DP ME dipole antennas with the property of a dynamic $H$-plane beamwidth control are achieved as shown in Figure 10 [14]. Two tunable parasitic dipoles are put on the sides of a driven ME dipole along its $H$-plane. Varactor diodes are loaded on the parasitic thin dipoles to change the strength of the mutual coupling. When changing the state of the varactor diode, the overall radiation pattern of the antenna could be tuned. Different from the first method, this design uses the magnitude distribution instead of the phase distribution in the first method to obtain beamwidth reconfiguration. Figure 11 describes the simplified equivalent circuit of the design. The mutual coupling between the driven and parasitic dipoles is represented by a transformer. Since the varactor diodes are inserted in the parasitic dipoles, the impedance of the parasitic element is able to be varied by means of changing the capacitance of the varactor diodes. Therefore, the magnitude and phase distribution of the driven and parasitic dipoles are decided by the varactor diodes. Consequently, the entire antenna works similarly to a threeelement array, where the center element is an ME dipole and the left and right elements are dipoles with an identical magnitude and phase distribution. The capacitance values of the varactor diodes determine the coupling strength and, in turn, the magnitude distribution. Hence, the entire radiation pattern of the antenna could be varied by tuning the power distribution of the dipoles. The simulated radiation patterns in the $H$-plane is presented in Figure 12 with different $C$ (the capacitance value of varactor diodes). We can see that, along with $C$ which reduces from 4 to $0.8 \mathrm{pF}$, the $3 \mathrm{~dB}$ beamwidth of the proposed antenna rises from $80^{\circ}$ to $160^{\circ}$.

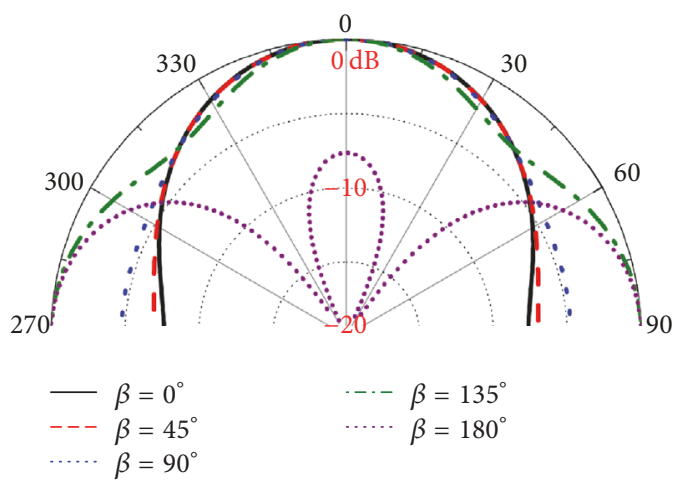

FIGURE 9: Array factor for different $\beta$ with $d=0.5 \lambda_{0}$ and $M=\sqrt{2}$.

Finally, an LP design with beamwidth reconfiguration is realized in [18]. As shown in Figure 13, the antenna is composed of a $\Gamma$-probe-fed ME dipole. Along its $H$-plane, there are three pairs of tunable strip gratings. Each strip is cut into 16 short portions and 15 PIN diodes are inserted into the gaps. When forward biased, the PIN diodes are ON and the strips serve as reflectors. When unbiased, the PIN diodes are OFF and the strips can be seen as transparent for radiating wave. Furthermore, because the PIN diodes on different strips are commanded by separated DC signals, by changing the amplitude of the DC signals, the size of the reflector can be varied as indicated in Figure 14. Therefore, the beamwidth of the proposed antenna could be changed. It should be emphasized that this method for beamwidth reconfiguration is different from the aforementioned. The first two methods are implemented by tuning the amplitude and phase distributions of the parasitic elements, while this design is realized by reconfiguring the size of the reflector. The characteristics of this simple antenna are highly attractive for applications in cellular systems. It has an impedance bandwidth as wide as $40 \%$. The $H$-plane beamwidth can be tuned from $153^{\circ}$ to $81^{\circ}$.

\section{Conclusion}

In this paper, some reconfigurable ME dipole antennas have been reviewed. A frequency reconfigurable design has been first reviewed, showing a wideband mode for sensing and reconfigurable narrowband modes for communications, which is attractive for base stations in cognitive radio. Secondly, polarization reconfigurable designs which are able to switch between the LP, RHCP, and LHCP states have been reviewed. The designs own the ability of degrading multipath-fading effects and enhancing the system stability and are useful for indoor wireless communication systems and can also be used as antenna elements for the outdoor base stations. Finally, three different beamwidth reconfigurable designs have been reviewed with dynamic control over their radiation beamwidth according to the environment requirement. These designs are attractive for outdoor base stations in future wireless communication systems.

Compared with other reconfigurable directional antenna candidates, the ME dipole owns some very attractive advantages as indicated in Table 1 . The ME dipole can obtain wide 


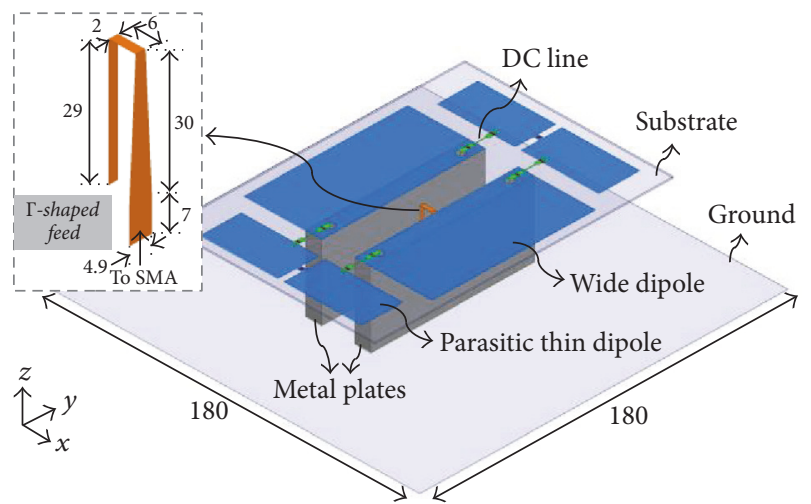

(a)

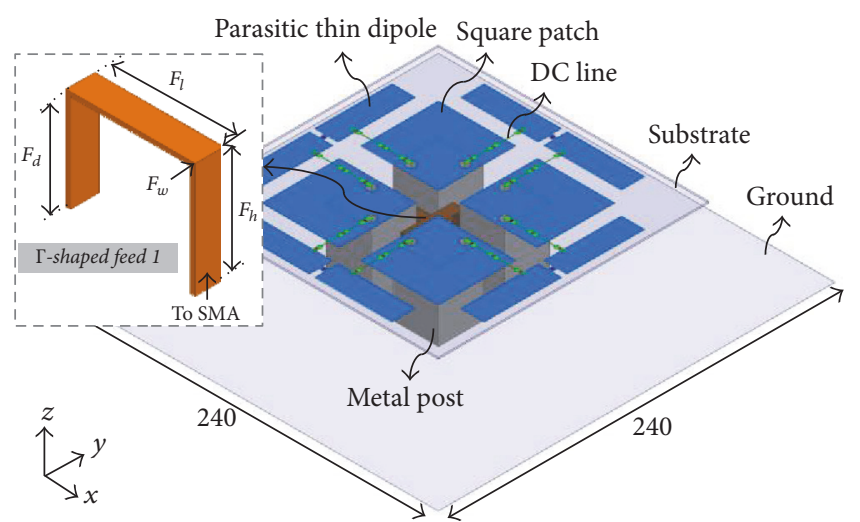

(b)

FIGURE 10: Geometry of the linearly polarized and dual-polarized beamwidth reconfigurable ME dipole antennas [14]: (a) LP antenna; (b) DP antenna.

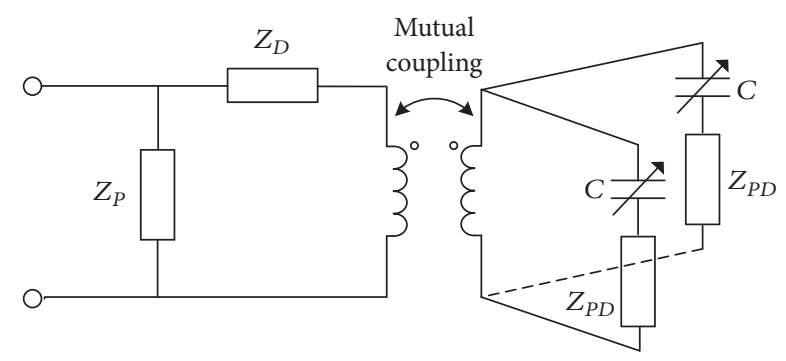

FIGURE 11: Equivalent circuit of the LP antenna. $Z_{D}, Z_{P}, Z_{P D}$ represent the impedances of the wide planer dipole, shorted quarterwavelength patch antenna, and the parasitic dipoles, respectively.

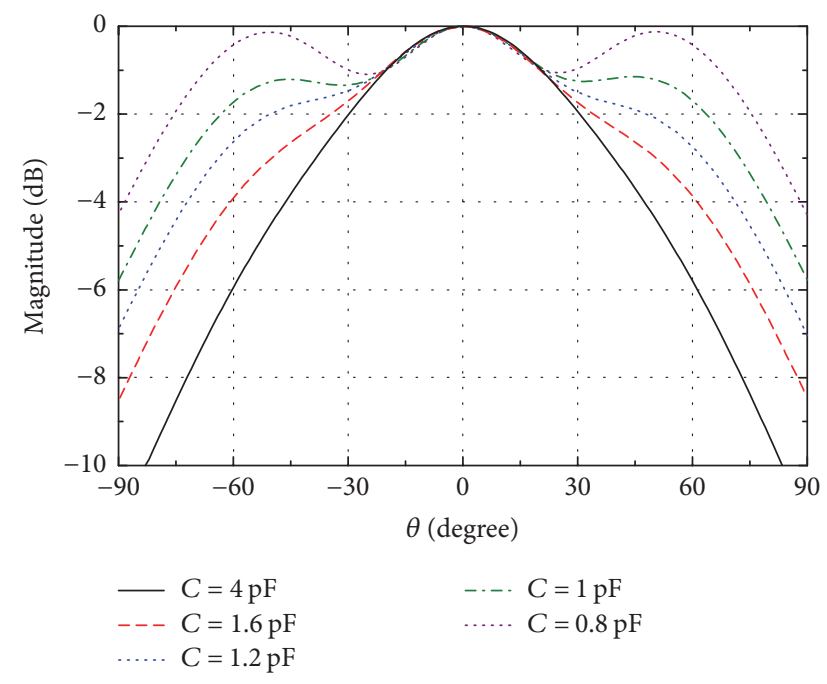

FIGURE 12: Simulated radiation patterns in the $H$-plane at $2 \mathrm{GHz}$ with different $C$.

bandwidth and excellent unidirectional radiation patterns when designed for reconfigurable antennas. This is attributed to the advantages of the ME dipole. Besides, since the DC biasing lines can be hidden in the metal posts (magnetic dipole), the DC biasing lines can cause ignorable effects on

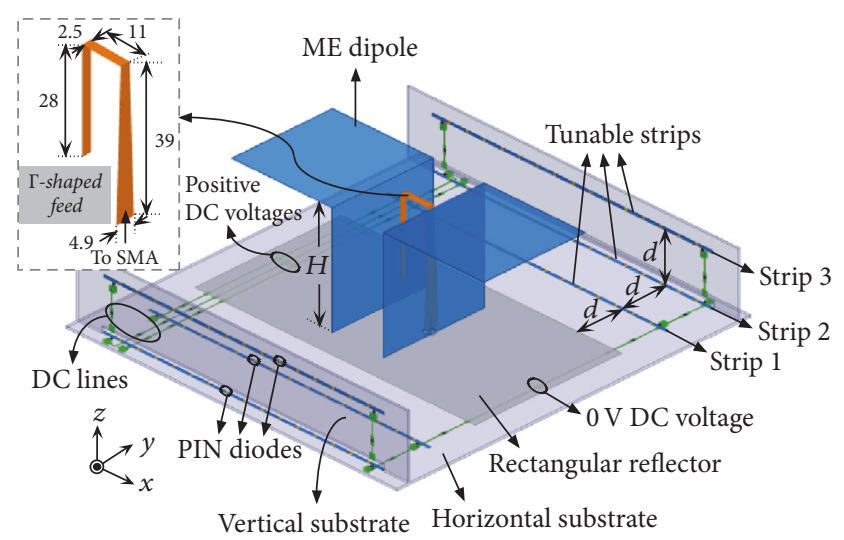

FIGURE 13: Geometry of the beamwidth reconfigurable ME dipole antenna using tunable strip grating.

TABLE 1: Comparison between ME dipole with other reconfigurable directional antenna candidates.

\begin{tabular}{lccc}
\hline $\begin{array}{l}\text { Reconfigurable } \\
\text { directional } \\
\text { antenna } \\
\text { candidates }\end{array}$ & Bandwidth & $\begin{array}{c}\text { Unidirectional } \\
\text { radiation } \\
\text { patterns } \\
\text { (front-to-back } \\
\text { ratio, } \\
\text { cross-pol) }\end{array}$ & $\begin{array}{c}\text { Difficulty level } \\
\text { to be } \\
\text { integrated with } \\
\text { tuning } \\
\text { mechanisms }\end{array}$ \\
\hline $\begin{array}{l}\text { Patch antenna } \\
{[1]}\end{array}$ & Narrow & Poor & Easy \\
\hline $\begin{array}{l}\text { Cavity-backed } \\
\text { slot antenna } \\
{[12]}\end{array}$ & Fair & Fair & Easy \\
\hline $\begin{array}{l}\text { Yagi-Uda } \\
\text { antenna } \\
{[13]}\end{array}$ & Fair & Fair & Difficult \\
\hline $\begin{array}{l}\text { Dipole } \\
{[2]}\end{array}$ & Wide & Good & Difficult \\
\hline \begin{tabular}{l} 
ME dipole \\
\hline
\end{tabular} & Wide & Excellent & Fair \\
\hline
\end{tabular}

the antenna performance. Therefore, the ME dipole is easy to be integrated with tuning mechanisms, especially with 


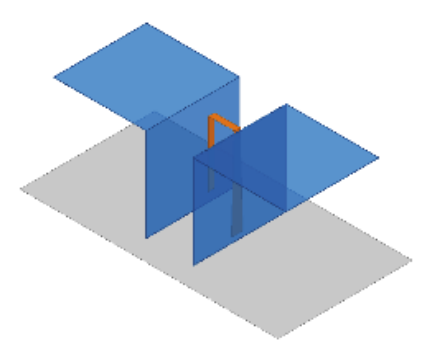

(a)

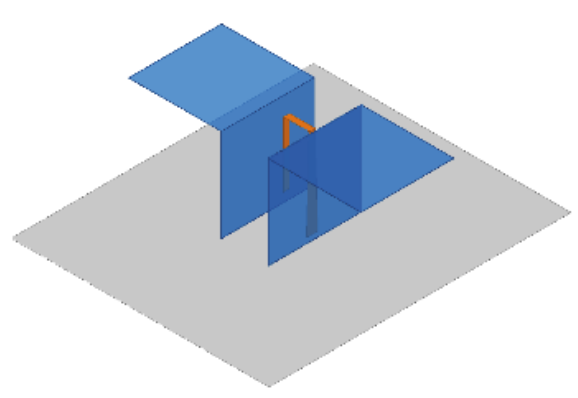

(c)

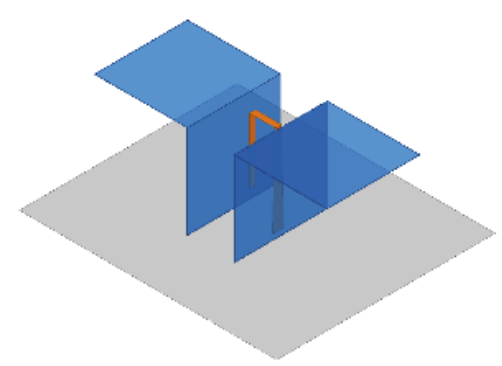

(b)

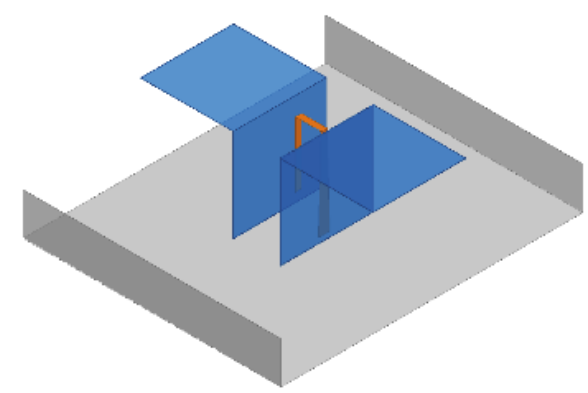

(d)

FIGURE 14: Equivalent structures of the antenna in different beamwidth states: (a) State 1; (b) State 2; (c) State 3; (d) State 4.

the electronically controlled switches. As discussed above, the ME dipole shows very attractive features over other directional antenna candidates in reconfigurable base station antenna design. These designs may be useful for base stations in wireless communication systems.

\section{Conflicts of Interest}

The authors declare that they have no conflicts of interest.

\section{Acknowledgments}

The work is supported by the National Natural Science Foundation of China (no. 61601303), Fundamental Research Foundation of Shenzhen (no. JCYJ20160308095149392), Fundamental Research Foundation of Shenzhen (no. JCYJ20170817095519575), and SZU R/D Fund (no. 2016022).

\section{References}

[1] M. R. Hamid, P. Gardner, P. S. Hall, and F. Ghanem, "Switchedband Vivaldi antenna," IEEE Transactions on Antennas and Propagation, vol. 59, no. 5, pp. 1472-1480, 2011.

[2] L. Ge and K.-M. Luk, "A band-reconfigurable antenna based on directed dipole," IEEE Transactions on Antennas and Propagation, vol. 62, no. 1, pp. 64-71, 2014.

[3] A. T. Kolsrud, M.-Y. Li, and K. Chang, "Dual-frequency electronically tunable CPW-fed CPS dipole antenna," IEEE Electronics Letters, vol. 34, no. 7, pp. 609-611, 1998.
[4] B. Avser and G. M. Rebeiz, "Tunable dual-band antennas for $0.7-1.1-\mathrm{GHz}$ and $1.7-2.3-\mathrm{GHz}$ carrier aggregation systems," Institute of Electrical and Electronics Engineers. Transactions on Antennas and Propagation, vol. 63, no. 4, part 2, pp. 1498-1504, 2015.

[5] K. Chung, Y. Nam, T. Yun, and J. Choi, "Reconfigurable microstrip-patch antenna with frequency and polarization-diversity functions," Microwave and Optical Technology Letters, vol. 47, no. 6, pp. 605-607, 2005.

[6] F. Wu and K.-M. Luk, "A Reconfigurable Magneto-Electric Dipole Antenna Using Bent Cross-Dipole Feed for Polarization Diversity," IEEE Antennas and Wireless Propagation Letters, vol. 16, pp. 412-415, 2017.

[7] F. Wu and K. M. Luk, "Wideband tri-polarization reconfigurable magneto-electric dipole antenna," Institute of Electrical and Electronics Engineers. Transactions on Antennas and Propagation, vol. 65, no. 4, pp. 1633-1641, 2017.

[8] L. Ge, X. Yang, D. Zhang, M. Li, and H. Wong, "PolarizationReconfigurable Magnetoelectric Dipole Antenna for 5G Wi-Fi," IEEE Antennas and Wireless Propagation Letters, vol. 16, pp. 1504-1507, 2017.

[9] W. Lin and H. Wong, "Polarization reconfigurable wheelshaped antenna with conical-beam radiation pattern," IEEE Transactions on Antennas and Propagation, vol. 63, no. 2, pp. 491-499, 2015.

[10] Y. J. Sung, T. U. Jang, and Y.-S. Kim, "A reconfigurable microstrip antenna for switchable polarization," IEEE Microwave and Wireless Components Letters, vol. 14, no. 11, pp. 534-536, 2004. 
[11] U. L. Bombale and S. Gupta, "Broadband planer array with switchable polarizations," Microwave and Optical Technology Letters, vol. 49, no. 10, pp. 2415-2419, 2007.

[12] J.-S. Row and M.-J. Hou, "Design of polarization diversity patch antenna based on a compact reconfigurable feeding network," IEEE Transactions on Antennas and Propagation, vol. 62, no. 10, pp. 5349-5352, 2014.

[13] P. F. Wahid, M. A. Ali, and B. C. DeLoach, "A reconfigurable Yagi antenna for wireless communications," Microwave and Optical Technology Letters, vol. 38, no. 2, pp. 140-141, 2003.

[14] L. Ge and K.-M. Luk, "Linearly polarized and dual-polarized magneto-electric dipole antennas with reconfigurable beamwidth in the H-plane," Institute of Electrical and Electronics Engineers. Transactions on Antennas and Propagation, vol. 64, no. 2, pp. 423-431, 2016.

[15] P.-Y. Qin, Y. J. Guo, A. R. Weily, and C.-H. Liang, "A pattern reconfigurable U-slot antenna and its applications in MIMO systems," IEEE Transactions on Antennas and Propagation, vol. 60, no. 2, pp. 516-528, 2012.

[16] G. Monti, L. Corchia, and L. Tarricone, "A microstrip antenna with a reconfigurable pattern for RFID applications," Progress in Electromagnetics Research B, no. 45, pp. 101-116, 2012.

[17] L. Ge and K. M. Luk, "A three-element linear magneto-electric dipole array with beamwidth reconfiguration," IEEE Antennas and Wireless Propagation Letters, vol. 14, pp. 28-31, 2015.

[18] L. Ge and K. M. Luk, "Beamwidth Reconfigurable MagnetoElectric Dipole Antenna Based on Tunable Strip Grating Reflector," IEEE Access, vol. 4, pp. 7039-7045, 2016.

[19] K. M. Luk and H. Wong, "A new wideband unidirectional antenna element," International Journal of Microwave and Optical Technology, vol. 1, no. 1, pp. 35-44, 2006.

[20] Y. Li and K. M. Luk, "60-GHz Dual-Polarized Two-Dimensional Switch-Beam Wideband Antenna Array of ApertureCoupled Magneto-Electric Dipoles," IEEE Transactions on Antennas and Propagation, vol. 64, no. 2, pp. 554-563, 2016.

[21] Y. Li and K.-M. Luk, "A 60-GHz wideband circularly polarized aperture-coupled magneto-electric dipole antenna array," Institute of Electrical and Electronics Engineers. Transactions on Antennas and Propagation, vol. 64, no. 4, pp. 1325-1333, 2016. 


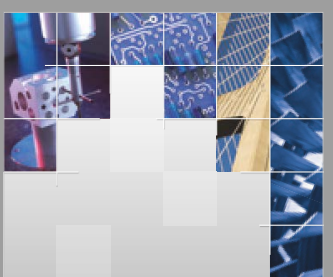

\section{Enfincering}
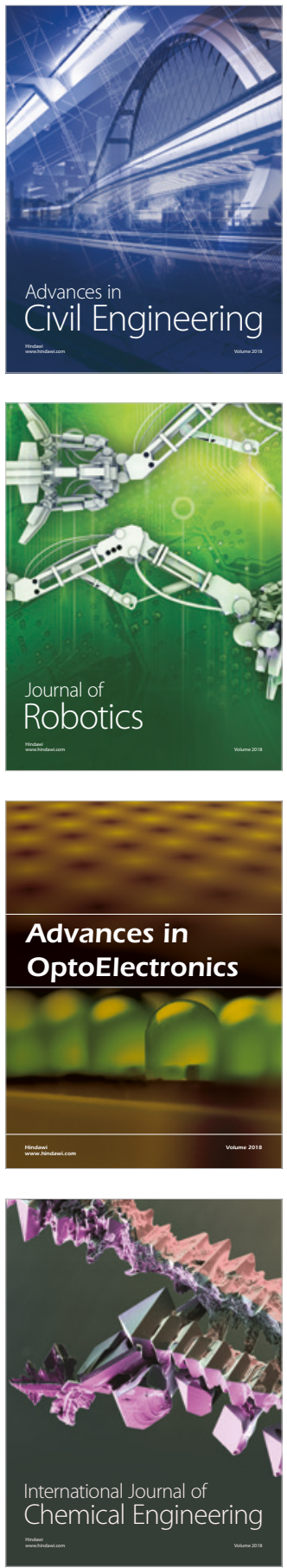

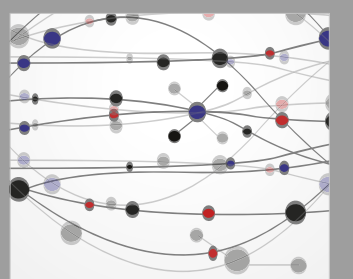

\section{Rotating \\ Machinery}

The Scientific World Journal

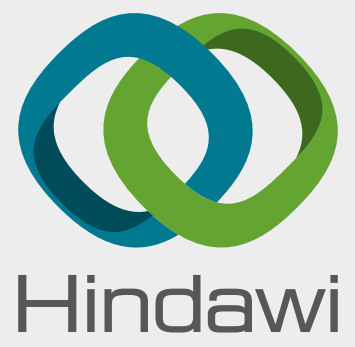

Submit your manuscripts at

www.hindawi.com
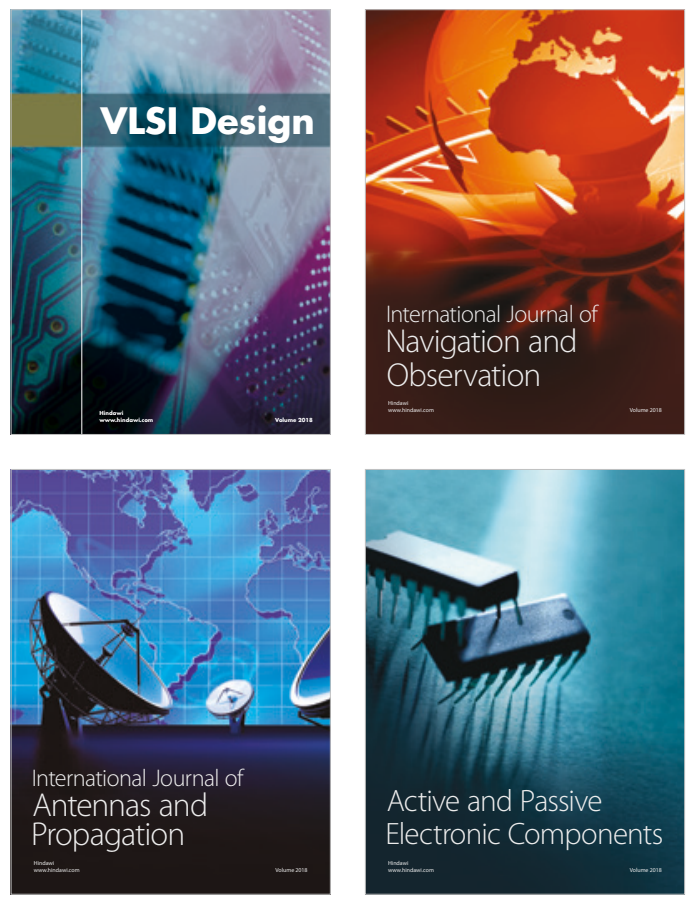
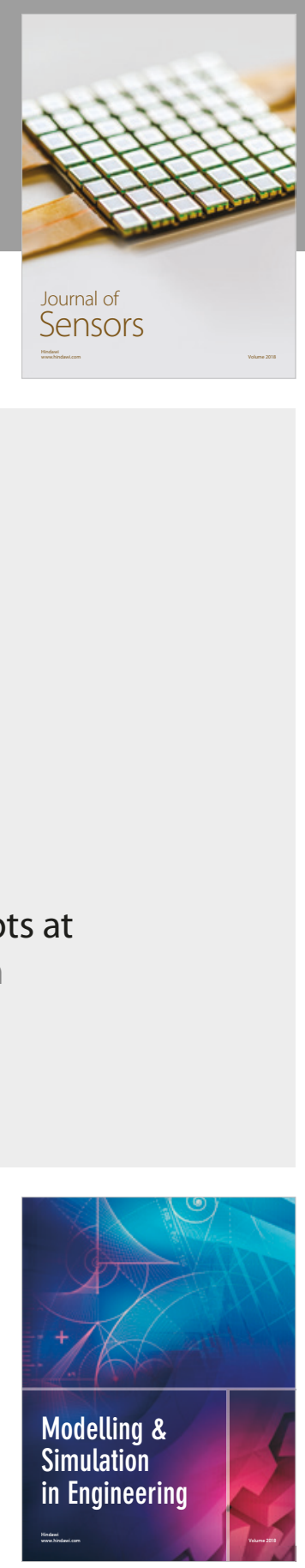

\section{Advances \\ Multimedia}
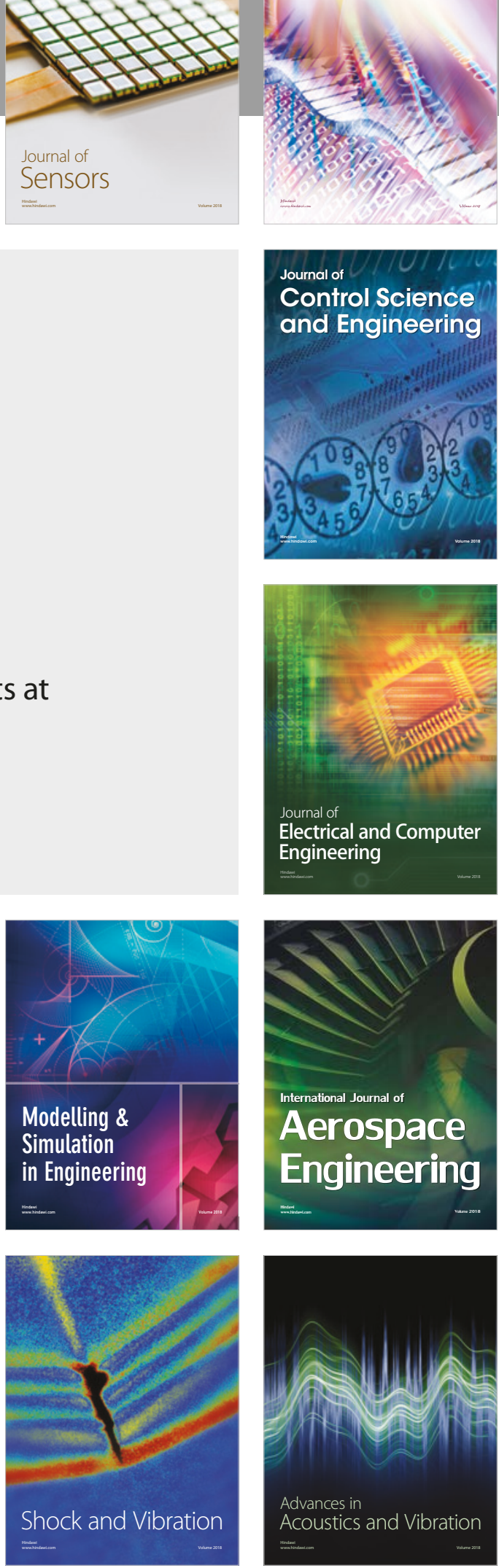